\title{
Strange results from chiral soliton models
}

\author{
Aleksey Cherman and Thomas D. Cohen \\ Department of Physics \\ University of Maryland \\ College Park, MD 20742
}

\begin{abstract}
The standard collective quantization treatment of the strangeness content of the nucleon in chiral soliton models such as the Skyrmion is shown to be inconsistent with the semi-classical expansion on which the treatment is based. The strangeness content vanishes at leading order in the semi-classical expansion. Collective quantization correctly describes some contributions to the strangeness content at the first nonvanishing order in the expansion, but neglects others at the same order - namely, those associated with continuum modes. Moreover, there are fundamental difficulties in computing at a constant order in the expansion due to the non-renormalizable nature of chiral soliton models. Moreover, there are fundamental difficulties in computing at a constant order in the expansion due to the non-renormalizable nature of chiral soliton models and the absence of any viable power counting scheme. We show that the continuum mode contribution to the strangeness diverges, and as a result the computation of the strangeness content at leading non-vanishing order is not a well-posed mathematical problem in these models.
\end{abstract}

The extraction of the "strangeness content of the nucleon" has been the subject of considerable experimental activity over the past twenty years, largely involving parity violating electron scattering 1]. One class of models studied extensively 2, 3, 4, 5, 6, 7, 8, 9, 10, 11, 12, 13, 14, 15, 16, 17, 18, 19, 20, 21] for the purpose of understanding the strangeness content (i.e. the matrix elements of strange quark bilinear operators in the nucleon) were chiral soliton models such as the Skyrmion 22]. The semi-classical treatment of these models based on collective quantization has provided a reasonably good description of many non-strange properties of the nucleon 23]. It is interesting to consider how well these models do in describing the strange matrix elements For example, the strange magnetic moment in these models typically comes out in the range $\mu_{s} \sim-0.5$ in units of the nuclear magneton with the strangeness radius of about $r_{s}^{2} \sim-0.1 \mathrm{fm}^{2}$ (with the exact values depending on the variant of the model used). The experimental values for these appear to be consistent with zero. A recent fit [24] to the world's data yields $\mu_{s}=0.12 \pm 0.55$ and $r_{s}^{s}=0.01 \pm 0.95 \mathrm{fm}^{2}$. Thus the values predicted in the models appear to be on the large size but do not appear to be inconsistent with the data.

What is one to conclude from this situation? Before this issue can be addressed, it is necessary to understand how these quantities were computed in these models. We note that these calculations have been based directly on collective quantization, or some variation of this such as the YabuAndo method 25] which reduces to collective quantization in the $S U(3)$ flavor limit. As will be shown in this letter, calculations of strange quark matrix elements in these models using collective quantization are inconsistent from the perspective of the semi-classical expansion, and hence should not be regarded as true predictions from the models. Moreover, the non-renormalizability of such models creates fundamen- tal difficulties intrinsic to any consistent description of the strangeness content.

These models are only known to be meaningful in the context of a semi-classical expansion. All treatments of the model begin with a classical solution which determines the gross structure of the baryon. To proceed in a systematic fashion, one must assume that a semi-classical treatment is valid. In this context, it will be shown that:

1. Strange quark matrix elements of the nucleon necessarily occur at a subleading order in a semi-classical expansion.

2. Certain subleading effects - those associated with the collective zero modes - are automatically included in treatments that use collective quantization. However, other contributions which also occur at the first nonvanishing order - those from the non-collective continuum modes - are not included. Since these effects have been neglected in typical computations of the strangeness content, the computations are inconsistent.

3. The computation of the strangeness content at leading non-vanishing order starting from the Lagrangian of the chiral soliton model in not a well-posed mathematical problem. Contributions from the continuum modes contribute at the leading non-vanishing order and are divergent. The models are not systematic effective field theories with controlled power counting; the divergences which appear cannot be absorbed by renormalizing coefficients in the models as they are given in their Lagrangians.

At first sight these results suggest a deficiency of Skyrmetype models for these observables. However, they actually 
highlight a strength: the ability of the models to encode the underlying quark-loop structure of QCD. The semi-classical expansion of the models corresponds to an expansion in the number of closed quark loops. At the QCD level, strange quark matrix elements come from quantum loops and thus have a qualitatively different origin than do non-strange matrix elements of the nucleon. It is a virtue of the semiclassical models that their structure forces one to impose additional physics inputs in the form of new prescriptions in order to describe the qualitatively distinct physics of the strangeness content of the nucleon.

The framework of the analysis is the semi-classical expansion. One natural way to justify it is via Witten's celebrated connection of the semi-classical treatment of the soliton with the large $N_{c}$ limit of QCD [26]; the semi-classical expansion of the soliton matches the $1 / N_{c}$ expansion of QCD. One need not invoke large $N_{c}$ as the ultimate justification of the semiclassical expansion. However, regardless of how of the expansion is justified, factors of $1 / N_{c}$ may be used as markers to keep track of orders in the semi-classical expansion. In the present context we work in the semi-classical analysis analogous to the standard large $N_{c}$ limit of 't Hooft 27], which is the appropriate limit when the usual $\mathcal{O}\left(N_{c}\right)$ strength is taken for the WWZ term 28].

To illustrate the underlying issues in a relatively simple context, we consider the strange scalar matrix element of the nucleon in the chiral limit of the three flavor version of Skyrme's original model[29]. However, the conclusions depend on neither the choice of observable or model. The action for the model is

$$
S=\int d^{4} x\left(\frac{f_{\pi}^{2}}{4} \operatorname{Tr}\left(L_{\mu} L^{\mu}\right)+\frac{\epsilon^{2}}{4} \operatorname{Tr}\left(\left[L_{\mu}, L_{\nu}\right]^{2}\right)+\frac{B_{0}}{4} \operatorname{Tr}\left(M\left(U+U^{\dagger}-2\right)\right)\right)+N_{c} S_{W W Z}
$$

where the left chiral current $L_{\mu}$ is given by $L_{\mu} \equiv U^{\dagger} \partial_{\mu} U$, with $U \in S U(3)_{f}\left[22\right.$, 30, 31]; $S_{W W Z}$ is the Witten-WessZumino (WWZ) term, whose inclusion is necessary for the Skyrme model to correctly encode the anomaly structure of QCD 28, 31]. The $U$ field can be written as $U=$ $\exp \left(i \sum_{a} \lambda_{a} \pi_{a} / f_{\pi}\right)$ where the $\pi$ are the Goldstone boson meson fields and the $\lambda_{a}$ are the Gell-Mann matrices; $M$ is the quark mass matrix. For simplicity the present analysis will be done in the chiral limit; however, the mass term is included as an external source. Thus the matrix element of interest - the strange scalar matrix element in the chiral limit - can be obtained by computing the nucleon mass for arbitrary values of the mass and then differentiating:

$$
\left\langle N\left|\bar{s} s-\langle\bar{s} s\rangle_{v a c}\right| N\right\rangle=\frac{d M_{N}}{d m_{s}}
$$

"vac" indicates a vacuum value.

At the QCD level, the strangeness content of the nucleon can only arise from quark loops. This already establishes point 1 above: there is a suppression factor of $1 / N_{c}$ for each quark loop. Thus strange quark matrix elements are subleading in the semi-classical expansion.

Consider the computation of the scalar strange quark matrix element using collective quantization, as done originally by Donahue and Nappi[32. The computation simplifies somewhat if one considers the ratio of the strange scalar matrix element to the total scalar matrix elements of the three light flavors; denote this ratio $X_{s}$ :

$$
X_{s} \equiv \frac{\left\langle N\left|\bar{s} s-\langle\bar{s} s\rangle_{\mathrm{vac}}\right| N\right\rangle}{\left\langle N\left|\bar{u} u+\bar{d} d+\bar{s} s-\langle\bar{u} u+\bar{d} d+\bar{s} s\rangle_{\mathrm{vac}}\right| N\right\rangle}
$$

$|N\rangle$ is the nucleon state. In collective quantization, the collective $\mathrm{SU}(3)$ rotation variables, $A$, act on the standard classical static hedgehog: $U=A^{\dagger} U_{h} A$ with the hedgehog Skyrmion defined as $U_{h} \equiv \exp (i \hat{r} \cdot \vec{\tau} f(r))$ (where $\vec{\tau}$ are the first three Gell-Mann matrices) ; $f(r)$ is the standard Skyrme profile function for states of baryon number $B=1$. Evaluating $X_{s}$ using standard $\mathrm{SU}(3)$ collective quantization [33] yields

$$
X_{s}=\frac{1}{3}\left\langle N\left|1-D_{88}\right| N\right\rangle=\frac{1}{3} \int d A \psi_{N}^{*}(A)\left(1-D_{88}\right) \psi_{N}(A)
$$

where $d A$ stands for the Haar measure on $\mathrm{SU}(3), D_{88}=$ $\frac{1}{2} \operatorname{Tr}\left[\lambda_{8} A \lambda_{8} A^{\dagger}\right]$ (which is an $\mathrm{SU}(3)$ Wigner D-matrix), and $\psi_{N}(A)$ is the collective wave function for the nucleon-i.e., an appropriately normalized SU(3) Wigner D-matrix. Evaluating the expression using the collective wave function for the nucleon gives 32 , 33] $X_{s}=7 / 30 \approx .23$. While this is relatively small numerically, it is non-zero. Note that this ratio does not depend on the form of the Skyrme profile function $f(r)$. This might suggest that it is a model-independent result, but as pointed out by Kaplan and Klebanov [34], this simple result is not universal and depends on the form of the mass term in the Skyrme lagrangian. 
Apparently the standard leading order collective quantization calculation used in the computation of strange quark matrix elements includes subleading effects in the semiclassical expansion. As was noted in ref. 35] one must include an explicit coefficient of $N_{c}$ rather than three as the coefficient of the WWZ term in order to make explicit the counting and trace orders in the semi-classical expansion. The coefficient of the WWZ term constrains the allowed $S U(3)$ multiplets. Thus at arbitrary $N_{c}$ the nucleon is in the generalized representation " 8 ", specified by $(p, q)=\left(1, \frac{N_{c}-1}{2}\right)[35] . \quad X_{s}$ can be computed at arbitrary $N_{c}$ 38 from Eq. (44) using standard group theoretical methods and the use of SU(3) Clebsch-Gordan coefficients appropriate for the "8" representation [36]. The result 34, 37, 38] is

$$
\begin{aligned}
& \left\langle N\left|\bar{s} s-\langle\bar{s} s\rangle_{\mathrm{vac}}\right| N\right\rangle^{\text {coll.quant. }} \\
= & \frac{2\left(N_{c}+4\right)}{N_{c}^{2}+10 N_{c}+21}\left\langle N\left|\bar{u} u+\bar{d} d+\bar{s} s-\langle\bar{u} u+\bar{d} d+\bar{s} s\rangle_{\mathrm{vac}}\right| N\right\rangle \\
= & \left(\frac{2}{N_{c}}+\mathcal{O}\left(1 / N_{c}^{2}\right)\right)\left\langle N\left|\bar{u} u+\bar{d} d-\langle\bar{u} u+\bar{d} d+\rangle_{\mathrm{vac}}\right| N\right\rangle .
\end{aligned}
$$

Clearly, $\left\langle N\left|\bar{s} s-\langle\bar{s} s\rangle_{\text {vac }}\right| N\right\rangle$ is subleading in $N_{c}$-and, hence, in the semi-classical expansion - as compared to its non-strange analog.

The collective quantization method builds in some contributions to $\left\langle N\left|\bar{s} s-\langle\bar{s} s\rangle_{v a c}\right| N\right\rangle$ at the leading nonvanishing order in the semi-classical expansion (i.e., the first subleading order). The question is whether it captures all of them. The answer is no: there are contributions to $\left\langle N\left|\bar{s} s-\langle\bar{s} s\rangle_{v a c}\right| N\right\rangle$ at first subleading order which are not included in the collective quantization. These may be computed via Eq. (2): one differentiates the first subleading contribution to the nucleon mass and with respect to $m_{s}$. The procedure for implementing the semi-classical expansion for the calculation of the mass of a topological soliton in a bosonic theory is very well established 39, 40, 41]: The boson fields are expanded around the classical solution to quadradic order and and then quantized. The next-to-leading contribution to the mass is simply the energy of the zero point motion of these harmonic modes.

In general there are contributions from both discrete eigenmodes and from continuum modes. The modes in the SU(3) Skyrme model around the standard hedgehog can be broken up into kaon modes and pion modes. From the structure of Eq. (11), it is clear that only the kaon modes depend on $m_{s}$ and contribute to the strangeness content. The kaon modes separate into modes carrying strangeness plus or minus one, corresponding to kaons and anti-kaons. Moreover, eigenmodes carry good total orbital angular momentum $L^{2}$ and good "grand spin" $\vec{g}=\vec{I}+\vec{L}$ with $g=L \pm 1 / 2$ [39, 40]. Thus, the contribution to the strangeness content at next- to-leading order (i.e. leading non-vanishing) in the semiclassical expansion is:

$$
\begin{aligned}
& \left\langle N\left|\bar{s} s-\langle\bar{s} s\rangle_{v a c}\right| N\right\rangle_{\mathrm{NLO}}= \\
& \frac{1}{2} \sum_{g, L}(2 g+1) \frac{d}{d m_{s}}\left(\sum_{n} \omega_{L g+; n}^{\mathrm{disc}}+\sum_{n} \omega_{L g-; n}^{\mathrm{disc}}\right)+ \\
& \frac{1}{2} \sum_{g, L}(2 g+1) \frac{d}{d m_{s}} \int \frac{d \omega}{\pi}\left(\delta_{L g+}^{\prime}(\omega)+\delta_{L g-}^{\prime}(\omega)\right) \omega
\end{aligned}
$$

where $\delta_{L g \pm}$ is the phase shift for given $L$, g-spin and strangeness \pm 1 and $\omega_{L g \pm ; n}^{\text {disc }}$ indicates the $n^{\text {th }}$ discrete frequency with fixed grand spin and strangeness.

The frequency of the discrete modes and the phase-shifts for the continuous modes can be computed from the equa(5) tions of motion for the kaon fluctuation around the soliton may be derived in the manner of Callan and Klebanov [39, 40]. A compact form for these is given in ref. 40]. The equations are naturally expressed in terms of dimensionless lengths and masses: $\tilde{r}=\frac{f_{\pi}}{\epsilon} r, \tilde{\omega}=\frac{\epsilon}{f_{\pi}} \omega$ and $\tilde{m}_{K}=\frac{\epsilon}{f_{\pi}} m_{K}$. (Note that the conventions used here differ from ref. [40]: the symbol $f_{\pi}$ here corresponds to $f_{\pi} / 2$ in ref. [40] and $\epsilon$ corresponds $\frac{1}{2 \sqrt{2} e}$ ). The equations for the modes are

$$
\left(y(\tilde{r}) \tilde{\omega}^{2} \mp 2 \lambda(\tilde{r}) \tilde{\omega}+\Theta\right) k_{l, g, \pm}^{\tilde{\omega}}(\tilde{r})=0
$$

with

$$
\Theta \equiv \tilde{r}^{-2} \partial_{\tilde{r}} h(\tilde{r}) \partial_{\tilde{r}}-\tilde{m}_{K}^{2}-V_{\mathrm{eff}}(\tilde{r}), \quad \lambda(\tilde{r}) \equiv-\frac{N_{c} f^{\prime} \sin (f)}{8 \pi^{2} \epsilon^{2} \tilde{r}^{2}},
$$

$y(\tilde{r}) \equiv 1+2 s(\tilde{r})+d(\tilde{r}), \quad h(\tilde{r}) \equiv 1+2 s(\tilde{r})$,

$d(\tilde{r}) \equiv f^{\prime 2}, \quad s(\tilde{r}) \equiv \sin ^{2}(f) / \tilde{r}^{2}, \quad c(\tilde{r}) \equiv \sin ^{2}(f / 2)$

where $f$ is the Skyrme profile, the prime indicates differentiation with respect to $\tilde{r}$ and

$$
\begin{aligned}
V_{\mathrm{eff}} & =-\frac{d+2 s}{4}-2 s(s+2 d) \\
& +\frac{(1+d+s)\left(L(L+1)+2 c^{2}+4 c \vec{I} \cdot \vec{L}\right)}{\tilde{r}^{2}} \\
& +\frac{6}{\tilde{r}^{2}}\left(s\left(c^{2}+(2 c-1) \vec{I} \cdot \vec{L}\right)+\partial_{\tilde{r}}\left((c+\vec{I} \cdot \vec{L}) f^{\prime} \sin (f)\right)\right) .
\end{aligned}
$$

In Eq. (7), the $\mp$ indicates the strangeness of the mode, $g$ the g-spin and $L$ the orbital angular momentum. Phase shifts may be extracted from the modes by comparing with solution for $f=0$.

It is known that there is only one discrete mode for this system [41]. The mode has $L=1, g=1 / 2$ and $s=-1$. Moreover, at $m_{s}=0$ the mode is collective and associated with flavor rotations out of the $\mathrm{SU}(2)$ subspace of the original 
hedgehog: $k_{L g s}^{\text {disc }}(r)=k_{1 \frac{1}{2}-}^{\text {disc }}(r)=\sin (f(r) / 2)$ where $k_{1 \frac{1}{2}-}^{\text {disc }}(r)$ is the spatial profile of the mode. By construction, at $m_{s}=0$ this collective mode is in a flat direction and has zero frequency. It makes a nonzero contribution in Eq. (77), however, since the derivative of the frequency with respect to $m_{s}$ is nonzero at $m_{s}=0$. The frequency of this mode can be expanded perturbatively in $m_{K}^{2}$ from the underlying equations of ref. [40]; one finds $\omega^{\text {disc }}=\frac{4 m_{K}^{2} f_{\pi}^{2}}{N_{c}} \int d^{3} x(1-\cos (f))$ which in turn implies that the discrete mode contribution to the strangeness content at first subleading order in the semi-classical expansion is:

$$
\left\langle N\left|\bar{s} s-\langle\bar{s} s\rangle_{\mathrm{vac}}\right| N\right\rangle^{\mathrm{disc}}=\frac{2}{N_{c}}\left\langle N\left|\bar{u} u+\bar{d} d-\langle\bar{u} u+\bar{d} d\rangle_{\mathrm{vac}}\right| N\right\rangle .
$$

As expected, the contribution from the discrete mode in Eq. (8) - associated with the collective motion in the flat direction - exactly reproduces the result of collective quantization given in Eq. (6) at first nonvanishing order in the semiclassical $\left(1 / N_{c}\right)$ expansion. However, the collective quantization does not include the contributions from the continuum modes. These contributions are clearly both nonzero generically - the phase shifts are nonzero and dependent on $m_{s}$ [40] - and are of the same order in the semi-classical expansion as the discrete mode contribution encoded in the collective quantization. Thus, calculations of the strangeness content which neglect these are unjustified from the perspective of the semi-classical expansion. Since these are neglected by those calculations on the market which purport to compute the strangeness in chiral solitons $2,3,3,4,5,6$, 7, 8, 9, 10, 11, 12, 13, 14, 15, 16, 17, 18, 19, 20, 21], one must regard these calculations as being inconsistent with the semi-classical expansion 42. This establishes point 2. We note that this point is implicit in the work of Kaplan and Klebanov 34].

The cure for this problem seems obvious: one ought to simply include these continuum mode contributions in the calculation. Unfortunately, the contribution from these modes diverges.

This divergence can be seen from the form of Eq. (7). For large $\omega$ and fixed $L$ the system is in the WKB regime. At $L=0$ and high $\omega$, a simple WKB calculation at lowest order in $\omega^{-1}$ yields that

$$
\begin{aligned}
\left.\frac{\partial \delta}{\partial m_{s}}\right|_{\left(m_{s}=0\right)} & =\frac{\alpha}{\omega}+\mathcal{O}\left(\omega^{-3}\right) \\
\text { with } \quad \alpha & =\frac{\epsilon}{f_{\pi}} \frac{d m_{K}^{2}}{d m_{s}} \int d \tilde{r} \frac{\tilde{r}}{2 \sqrt{h(\tilde{r}) y(\tilde{r})}} .
\end{aligned}
$$

In fact, Eq. (9) holds for all partial waves (although the value of $\omega$ at which the asymptotic regime sets in grows with $L$ ). The reason for this is that although the angular momentum potential barrier grows with $L$, at any fixed $L$ we are free to choose an arbitrarily high $\omega$. This allows the WKB region to penetrate close to the origin, where the sum of the in- and out-going wave functions must vanish as a boundary condition, just as it does for $L=0$. Recalling Eq. (77) one sees from the above that the continuum mode sum contributions in all channels diverge logarithmically in the ultraviolet limit in a universal way. Thus the continuum mode contribution to the strangeness content of the nucleon is divergent. This divergence is not surprising: it reflects the one-loop divergences present in the underlying mesonic theory.

To make meaningful predictions from the theory, one must render this divergence finite in a manner consistent with the theory. If the theory were renormalizable this would be a well-defined task; any divergence which arises in the loops could be absorbed by renormalization of the constants in the original theory. However, chiral soliton models such as the Skyrme model are not renormalizable. More significantly, chiral soliton models are not effective field theories since they lack a systematic power counting scheme. Terms with any number of derivatives of the meson fields contribute at every order in the $1 / N_{c}$ expansion. Thus one cannot use power counting to restrict the number and type of counterterms at next-to-leading order in the semi-classical expansion: one needs an entirely ad hoc and uncontrolled prescription.

Of course, the act of building a chiral soliton model in the first place required making a similarly bold prescription: of the infinite number of terms which could be included at leading order in the $1 / N_{c}$ expansion only a very few are kept. The troubling issue here, however, is that unlike for leading order observables, the initial prescription used to set up the model is not sufficient to compute strange quark matrix elements; an additional prescription is needed. Since the computation of all strange quark matrix elements completely depends on the prescription used at next-to-leading order, the initial model given by the lagrangian, on its own, has no predictive power for these matrix elements.

This implies that the problem of computing the strangeness content from chiral soliton models at the first nonvanishing order in the semi-classical approximation is not well-posed. To proceed, one must make some prescription not fixed from the Lagrangian of the original model. This is highly problematic in that result for the strange content is not fixed by the original model. This establishes our final point. As we noted above, this is unsurprising: the strangeness content arises from quark loops. This is qualitatively different from the dominant origin of non-strange matrix elements and thus new physical inputs are required.

It should be clear that the conclusions in this letter are quite general. Although we have focused on the problem of computing the scalar matrix element at zero momentum transfer at the chiral limit of the Skyrme model, the structure of the argument holds quite generally. The argument 
that the contributions come from quark loops and must be subleading in the semi-classical expansion holds generally for any strange operator in any model and regardless of whether the system is in the chiral limit. We have shown this explicitly for the scalar matrix elements in the Skyrme model by demonstrating that the collective quantization leads to contributions which are subleading in $1 / N_{c}$ (and hence in the semi-classical expansion). We have explicitly verified that the same thing occurs for the case of the strange electric form factor - as it must. The general argument that the quantum fluctuations of all modes, collective and noncollective alike, contribute at the lowest nonvanishing order in the semi-classical expansion again holds for any strange matrix element in any model whether in the chiral limit or not. The need for a prescription not contained in the original model to compute at the leading nonvanishing order in the semi-classical expansion also applies to all strange quark observables in any non-renormalizable chiral soliton model.

\section{Acknowledgments}

This work is supported by the U. S. Department of Energy under grant number DE-FG02-93ER-40762. We are grateful to David Kaplan, Igor Klebanov, and Victor Kopeliovich for helpful discussions.
[1] For recent reviews see A. Acha et al., HAPPEX collaboration, arXiv:nucl-ex/0609002 ; E.J. Beise, M.L. Pitt and D.T. Spayde, Prog. Part. Nucl. Phys. 54, 289 (2005); D.H. Beck and R.D. McKeown Annu. Rev. NuclPart. Sci. 51, 189 (2001); D.H. Beck and B.R. Holstein, Int. Jour. Mod. Phys. E10, 1 (2001).

[2] V. B. Kopeliovich, J. Exp. Theor. Phys. 85, 1060 (1997) arXiv:hep-th/9707067.

[3] H. Weigel, UNITU-THEP-5-1997, presented at International Workshop on Strange Structure of the Nucleon, Geneva, Switzerland, 11-15 Mar 1997

[4] M. Wakamatsu, Phys. Rev. D 54, 2161 (1996).

[5] C. V. Christov et al., Prog. Part. Nucl. Phys. 37, 91 (1996) arXiv:hep-ph/9604441.

[6] J. R. Ellis and M. Karliner, arXiv:hep-ph/9601280.

[7] H. Weigel, Int. J. Mod. Phys. A 11, 2419 (1996) arXiv:hep-ph/9509398.

[8] H. C. Kim, A. Blotz, C. Schneider and K. Goeke, Nucl. Phys. A 596, 415 (1996) arXiv:hep-ph/9508299.

[9] J. Kroll and B. Schwesinger, Phys. Lett. B 334, 287 (1994) arXiv:hep-ph/9405267.

[10] H. Weigel, arXiv:hep-ph/9211259

[11] M. Karliner, TAUP-1932-91, invited talk at Int. Symp. on pi $N$ Physics and the Structure of the Nucleon, Bad Honnef, Germany, Sep 9-13, 1991

[12] N. W. Park and H. Weigel, Nucl. Phys. A 541, 453 (1992).

[13] M. Praszalowicz, Nuovo Cim. A 102, 39 (1989).

[14] D. Klabucar and I. Picek, Nucl. Phys. A 514, 689 (1990).

[15] N. W. Park, J. Schechter and H. Weigel, Phys. Rev. D 41, 2836 (1990).

[16] H. Weigel, J. Schechter, N. W. Park and U. G. Meissner, Phys. Rev. D 42, 3177 (1990).

[17] N. W. Park, J. Schechter and H. Weigel, Phys. Lett. B 224, 171 (1989).

[18] V. Bernard and U. G. Meissner, Phys. Lett. B 216, 392 (1989).

[19] V. M. Khatsimovsky, I. B. Khriplovich and A. R. Zhitnitsky, Z. Phys. C 36, 455 (1987).

[20] S. J. Brodsky, J. R. Ellis and M. Karliner, Phys. Lett. B 206, 309 (1988).
[21] C. W. Wong, D. Vuong and K. c. Chu, Nucl. Phys. A 515, 686 (1990).

[22] T. H. Skyrme, Proc. Roy. Soc. A260, 127 (1961).

[23] I. Zahed and G. E. Brown, Phys. Rept. 142, 1 (1986).

[24] R. D. Young, J. Roche, R. D. Carlini and A. W. Thomas, Phys. Rev. Lett. 97, 102002 (2006).

[25] H. Yabu and K. Ando, Nucl. Phys. B 301, 601 (1988).

[26] E. Witten, Nucl. Phys. B 160, 57 (1979).

[27] G. 't Hooft, Nucl. Phys. B 72, 461 (1974).

[28] E. Witten, Nucl. Phys. B 223, 422 (1983).

[29] E. Guadagnini, Nucl. Phys. B 236, 35 (1984).

[30] G. S. Adkins, C. R. Nappi and E. Witten, Nucl. Phys. B228 (1983) 552.

[31] E. Witten, Nucl. Phys. B 223, 433 (1983).

[32] J.F. Donoghue and C. R. Nappi, Phys. Lett. 168B, 105 (1986).

[33] J. Schechter and H. Weigel, arXiv:hep-ph/9907554

[34] D. B. Kaplan and I. R. Klebanov, Nucl. Phys. B 335, 45 (1990).

[35] T. D. Cohen, Phys. Lett.581B, 175 (2004); Phys. Rev. D70, 014011 (2004).

[36] T. D. Cohen and R. F. Lebed, Phys. Rev. D 70, 096015 (2004).

[37] V. B. Kopeliovich, Phys. Part. Nucl. 37, 623 (2006) arXiv:hep-ph/0507028.

[38] A. Cherman and T. D. Cohen, Phys. Lett. B 641, 401 (2006) arXiv:hep-th/0607110.

[39] C. G. . Callan and I. R. Klebanov, Nucl. Phys. B 262, 365 (1985).

[40] I. R. Klebanov, PUPT-1158, lectures given at NATO ASI on Hadron and Hadronic Matter, Cargese, France, Aug 8-18, 1989

[41] N. Itzhaki, I. R. Klebanov, P. Ouyang and L. Rastelli, Nucl. Phys. B 684, 264 (2004) arXiv:hep-ph/0309305.

[42] Ref. 21] is an exception and does discuss the mode sums. However it does so in the context of the kaon number, an unphysical quantity, which is related to the strangeness content via an uncontrolled approximation. 